Diabetologia 4, 365-369 (1968)

\title{
Einfluß der Prednisolonbehandlung auf die Insulinsekretion der Ratte
}

\author{
J. Marco*, F. Melani, R. Goberna**, W.H. Rott und E.F. Pfeiffer**** \\ Abteilung für Endokrinologie und Stoffwechsel (Prof. Dr.PFELFER) des Zentrums für Innere Medizin dex Universität \\ Ulm (Medizinisch-Naturwissenschaftliche Hochschule) \\ Eingegangen am 4. April 1968
}

\begin{abstract}
Effect of Treatment with Prednisolone on the Secretion of Insulin in Rats

Summary. The relationship between immunologically measurable insulin (IMI) in serum and the blood sugar was studied in the fasting state as well as after i.v. injection of glucose $(50 \mathrm{mg} / 100 \mathrm{~g} \mathrm{rat})$, in a group of untreated rats and in three groups of rats treated subcutaneously with $10 \mathrm{mg}$ Prednisolone daily for 3, 7 and 18 days, resp. Only in the group of rats treated with Prednisolone for 7 days was there a significant increase in the fasting blood sugar; in all of the treated groups the glucose assimilation coefficient $(\mathrm{K})$ remained within the normal range. The fasting insulin levels, however, showed a significant rise compared with the control group. After glucose loading in all the treated groups comparatively higher insulin values in blood were also observed. - This hyperinsulinism does not seem to be a result of hyperglycaemia, and is accompanied by neither a decreased assimilation of glucose nor an increase in the reactivity of the endocrine pancreas to glucose. Our findings are an indication of the existence of a peripheral antagonism between glucocorticoids and in sulin, which leads to an increased secretion of insulin in order to maintain the equilibrium of the carbohydrate metabolism.
\end{abstract}

L'effet du traitement à la prednisolone sur la secrétion d'insuline chez le rat

Resumé. Les auteurs ont étudié chez des rats traités à la prednisolone après l'injection intraveineuse de glucose (50 mg/100 g poids) le comportement du glucose du sang et de l'insuline immunoréactive du sérum. Trois groupes d'animaux ont reçu $10 \mathrm{mg}$ d'Ultracorten-H par jour par voie souscutanée, l'un pendant 3 jours, l'autre pendant 7 jours et le troisième pendant 18 jours. En comparaison avec un groupe de rats non traités, seuls les rats traités pendant 7 jours ont montré une augmentation significative de la glycémie à jeun. Le coefficient d'assimilation glucidique restait dans les limites normales dans les trois groupes d'animaux traités. Mais dans ces trois groupes traités à la prednisolone on a trouvé des taux élevés d'insuline sérique à jeun et aussi une sécrétion d'insuline plus intense pendant l'épreuve d'hyperglycémie i.v. en comparaison avec le groupe de contrôle. Cet hyperinsulinisme ne semble pas être le résultat d'une hyperglycémie et n'est accompagné ni d'une réduction de l'assimilation du glucose ni d'une réactivité augmentée du pancréas endocrine envers le glucose. Nos résultats semblent indiquer un antagonisme périphérique entre les glucocorticoïdes et l'insuline, qui conduit à une sécrétion augmentée d'insuline pour maintenir l'équilibre du métabolisme glucidique.

Zusammenfassung. Bei mit Prednisolon behandelten Ratten wurde das Verhalten von Blutzucker und immunologisch meßbarem Insulin (IMT) im Serum nach i.v. Glucosebelastung ( $50 \mathrm{mg} / 100 \mathrm{~g}$ Körpergewicht) untersucht. Drei Tiergruppen erhielten über einen Zeitraum von 3, 7 bzw. 18 Tage $10 \mathrm{mg} /$ die Ultracorten-H subcutan. Im Vergleich zu einer Gruppe unbehandelter Kontrolltiere zeigten nur die Ratten des 7-Tage-Versuchs eine signifikante Erhöhung des Nüchternblutzuckers. Der Glucoseassimilations-Koeffizient $(K)$ blieb bei allen drei behandelten Gruppen im Normbereich. Jedoch war bei allen drei Gruppen der Prednisolon-behandelten Tiere im Vergleich zur Kontrollgxuppe sowohl ein erhöhter Nüchterninsulinspiegel als auch eine vermehrte Insulinsekretion nach der Glucosebelastung zu beobachten. Dieser Hyperinsulinismus scheint keine Folge einer Hyperglykämie zu sein und wird weder von einer Verminderung der Glucoseassimilation noch von einer verstärkten Reaktivität des endokrinen Pankreas gegenüber Glucose begleitet. Unsere Befunde sprechen für einen peripheren Antagonismus zwischen den Glucocorticoiden und Insulin, der, um den Kohlenhydratstoffwechsel im Gleichgewicht zu halten, zu einer vermehrten Insulinsekretion führt.

Key-words: Prednisolone, rat, glucose, immunologically measurable insulin (IMI), glucose assimilation coefficient (K), correlation, regression coefficient.
Die Erzeugung eines diabetischen Zustandes durch Verabreichung pharmakologischer Glucocorticoiddosen gelang zum ersten Mal Ira.Le (1941) bei Ratten, die zwangsweise mit einer kohlenhydratreichen Diät gefüttert wurden. Andere Autoren konnten den Versuch reproduzieren, indem sie die hyperglykämische Wirkung der Glukocorticoide durch Induzierung einer subdiabetischen Stoffwechsellage mittels vorheriger

* Stipendiat der Alexander von Humboldt Stiftung, Bad Godesberg.

** Stipendiat des Ministerio de Educación y Ciencia, Spanien.

*** Durchgeführt mit Unterstützung der Deutschen Forschungsgemeinschaft, Bad Godesberg. partieller Pankreatektomie (INGLI u. ThonN, 1941), Verabreichung kleiner Mengen von Alloxan (ZuokeR, 1949) oder Zugabe von Dehydroascorbinsäure bzw. Glutathion (LAZAROW, 1952) steigerten. Im gleichen Versuchstier erzeugten Franckson et al. (1953) einen „Steroid-Diabetes" allein durch 19-tägige Applikation von $2.5 \mathrm{mg} /$ die Cortison pro $100 \mathrm{~g}$ Ratte.

Andererseits ist bekannt, daß nach Glucocorticoidbehandlung das histologische Bild des Pankreas auf eine Ưberaktivität des Inselsystems hinweist (KINASH u. Harst, 1954; VolK u. Lazarus, 1963; VRanio, 1965). Gleichfalls konnten sowohl beim Cushing-Syndrom (Schwarz et al., 1962; MeLant (unveröffentlichte Befunde); KIINK u. EsTRICH, 1964; KarAM et al., 1965), 
als auch nach Glucocorticoidtherapie hohe Insulinwerte im Blut beobachtet werden. So fanden PerLEX und KrPnis (1966) beim Menschen nach Verabreichung von Dexamethason erhöhte Nüchterninsulinspiegel (IMI) und übernormale Insulinwerte nach i.v. Injektion von Tolbutamid und nach oraler Gabe von Glucose. CAMPBinL et al. (1966) berichteten über einen Anstieg der Nüchterninsulinspiegel (IMI) beim Chinesischen Hamster nach Cortisonbehandlung. BAssET u. WALLACE (1967) beschrieben bei Cortisol-behandelten Schafen eine Erhöhung des Nüchterninsulinspiegel (IMI) und eine verstärkte Insulinsekretion nach Futterzufuhr und nach i.v. Glucosebelastung. Unter ähnlicher Behandlung beobachteten WuLIIAMsoN (1962) bei der intakten Ratte eine Erhöhung der Insulinähnlichen Aktivität (ILA) im Plasma und SoTrer (1967) bei der adrenalektomierten Ratte eine Zunahme des ,nicht hemmbaren Insulins" (NSILA).

In der vorliegenden Arbeit haben wir an der Ratte den Einfluß der Prednisolonbehandlung auf das Verhalten von immunologisch meßbarem Insulin (IMI) und Glucose im Blut im Nïchternzustand sowie nach i. v. Glucosebelastung untersucht.

\section{Untersuchungsmethoden}

Bei allen Versuchen wurden männliche Ratten (Wistar WU) mit einem Gewicht um $300 \mathrm{~g}$ verwendet. Die Tiere erhielten eine pelletierte Standardkost (Altromin-R, B 0100) und Trinkwasser ad libitum. Vor der Belastung, die morgens um 8 Uhr vorgenommen wurde, hungerten die Ratten 12 Std lang. 20 Min. nach Nembutal-Betäubung (5 mg / $100 \mathrm{~g}$ Körpergewicht, i.p.) wurde die Glucose während einer Minute in die Vena femoralis injiziert $(50 \mathrm{mg} / 100 \mathrm{~g}$ Körpergewicht, aus einer 10\% igen Lösung). Die Blutentnahme erfolgte nach Decapitation im Nüchternzustand und 5, 15, 30, 45 und 60 Min. nach Glucosezufuhr. Wegen der Schwierigkeit, eine ausreichende Blutmenge für die Zucker- und IMI-Bestimmung zu gewinnen, verwendeten wir 1 Ratte für jeden Wert; für eine vollständige Belastung benötigten wir somit 6 Tiere.

Der Versuchsplan und die Zahl der Tiere für jeden Wert und Versuch gehen aus folgender Aufstellung hervor:

\begin{tabular}{lrrrrrr}
\hline & 0 & 5 & 15 & 30 & 45 & $60 \mathrm{Min}$. \\
\hline unbehandelte Ratte & 13 & $\mathbf{1 3}$ & $\mathbf{1 3}$ & $\mathbf{1 3}$ & 13 & $\mathbf{1 3}$ \\
3-Tage-Behandlung & 12 & $\mathbf{1 2}$ & $\mathbf{1 2}$ & $\mathbf{1 2}$ & 12 & $\mathbf{1 2}$ \\
7-Tage-Behandlung & 12 & 11 & $\mathbf{1 1}$ & 11 & $\mathbf{1 1}$ & $\mathbf{1 1}$ \\
18-Tage-Behandlung & $\mathbf{9}$ & 8 & $\mathbf{9}$ & $\mathbf{9}$ & $\mathbf{9}$ & $\mathbf{9}$ \\
\hline
\end{tabular}

Das Prednisolon (Ultracorten-H wasserlöslich, CIBA) wurde in aqua bidest. gelöst $(5 \mathrm{mg} / \mathrm{ml})$ und $10 \mathrm{mg} /$ die, aufgeteilt in zwei Dosen von je $5 \mathrm{mg}$ morgens und abends, subcutan verabreicht.

Der Blutzucker wurde reduktometrisch mit dem Technicon Auto-Analyzer bestimmt. Den Urin untersuchten wir mit Glukotest-Boehringer, Mannheim, auf
Glucose. Die gewonnenen Seren zur Insulinbestimmung wurden bei $-20^{\circ} \mathrm{C}$ aufbewahrt und am Ende des ganzen Versuchs in einem Ansatz nach MeLANI et al. (1965) gemessen. Freies und gebundenes Insulin- ${ }^{131} \mathrm{Jod}$ trennten wir mit Hilfe von Amberlite CG 400 I. Für die Aufstellung der Eichkurve und für die Markierung mit ${ }^{131} \mathrm{Jod}$ verwendeten wir Schweineinsulin $(25 \mathrm{E} / \mathrm{mg}$ NOVO, Kopenhagen).
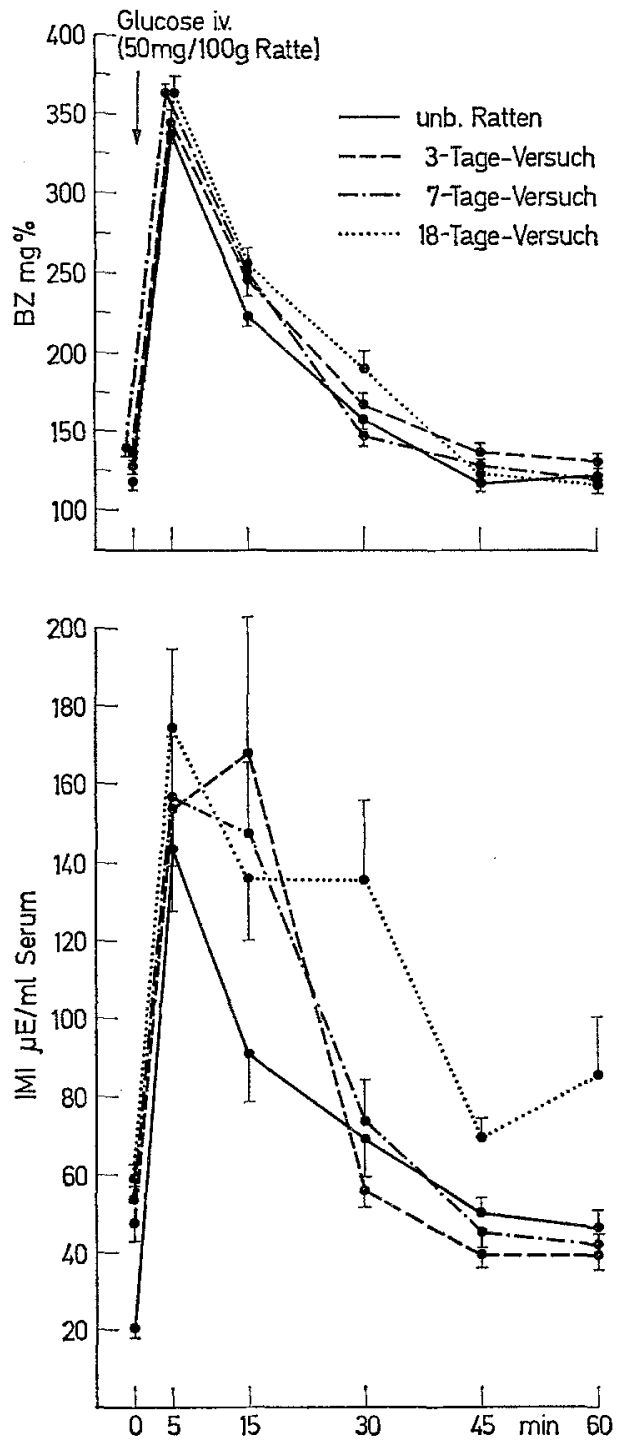

Abb. 1. Blutzucker und immunologisch meßbares Insulin (IMI) im Serum im Nüchternzustand und nach i.v. Glucosebelastung bei unbehandelten sowie bei 3,7 und 18 Tage lang mit $10 \mathrm{mg} /$ die Prednisolon behandelten Ratten. SEM

\section{Ergebnisse}

In Abb. 1 sind die Glucose- und Insulin-Kurven jeder Gruppe dargestellt.

Glucose. Vergleicht man die einzelnen Werte der drei Behandlungsgruppen mit den entsprechenden Werten der Kontrollreihe, so zeigt sich folgendes Bild: 
Beim 3-Tage-Versuch ist der 45-Minuten-Wert signifikant erhöht $(p<0.005)$; beim 7-Tage-Versuch läßt sich eine Erhöhung des Nüchternblutzuckers $(p<$ $0.05)$, sowie der 5-Minuten- $(p<0.02)$, 15-Minuten$(p<0.05)$ und 45-Minuten-Werte $(\mathrm{p}<0.05)$ statistisch sichern; beim 18-Tage-Versuch ist lediglich der 30-Minuten-Wert signifikant erhöht $(p<0.02)$. Der Glucoseassimilations-Koeffizient (K), nach CoNARD (1955) berechnet, wird jedoch von der Glucocorticoidbehandlung nicht beeinflußt; er liegt bei allen untersuchten Gruppen im Normbereich und ist von derselben Größenordnung wie der aus den Befunden von Noweld u. Howland (1966) bei normalen Ratten berechnete Wert (Tabelle 1). Bei täglicher Kontrolle wurde in keinem Fall eine Glucosurie beobachtet.

Tabelle 1. Glucoseassimilations-Koeffizient $(K)$ und Nüchtern-Insulin-Werte (IMI, Mittelwerte $\pm S E M)$ der verschiedenen Versuchsgruppen

\begin{tabular}{lll}
\hline Gruppe & $\mathrm{K} \cdot 10^{-2}$ & $\begin{array}{l}\text { Nüchtern- } \\
\text { IMI-Werte } \\
(\mu \mathrm{E} / \mathrm{ml} \text { Serum) }\end{array}$ \\
\hline unbehandelte Ratten & 2.17 & $21 \pm 2.19$ \\
3-Tage-Versuch & 1.95 & $48 \pm 4.86$ \\
$\begin{array}{l}\text { 7-Tage-Versuch } \\
\text { 18-Tage-Versuch }\end{array}$ & 2.15 & $59 \pm 4.22$ \\
$\begin{array}{l}\text { Normale Ratten; aus } \\
\text { den Werten von NowEL } \\
\text { u. HowLAND (1966) }\end{array}$ & 2.36 & $54 \pm 4.82$ \\
\hline
\end{tabular}

Insulin. Bei den drei mit Prednisolon behandelten Gruppen stieg der Nüchterninsulinspiegel im Blut bemerkenswert - auf mehr als das Doppelte - an $(p<$ 0.001 ). Ebenso findet man bei diesen drei Gruppen 15 Min. nach Glucosegabe höhere IMI-Werte $(p<$ $0.05 ; p<0.02) p<0.05$ ). Im 18-Tage-Versuch manifestiert sich der Hyperinsulinsmus auch 30 Min. $(p<$ $0.005)$, 45 Min. $(p<0.005)$ und 60 Min. $(p<0.02)$ im Verlauf der Belastung.

Gewicht. Unter der Prednisolonbehandlung wurde bei den Ratten des 7-Tage-Versuchs eine Gewichtsabnahme von $5 \%$ und bei denen des 18-Tage-Versuchs von $12 \%$ festgestellt. Beim 3-Tage-Versuch war keine Änderung des Gewichts nachzuweisen.

\section{Diskussion}

Wie schon einleitend erwähnt, konnten FrancKsov et al. (1953) bei Ratten allein durch die Verabreichung von Cortison einen diabetischen Zustand hervorrufen. Obwohl wir bei unseren Versuchen Prednisolon verwendeten, das auf den Kohlenhydratstoffwechsel 5 mal stärker wirkt als Cortison (WESr, 1957) und außerdem eine höhere Dosis injizierten, zeigten sich nur beim 7-Tage-Versuch Anzeichen einer diabetischen Stoffwechsellage im Sinne eines erhöhten Nüchtern- blutzuckers. Bei allen drei behandelten Gruppen blieb der Koeffizient der Glucoseassimilation (K) im Bereich der Norm, und es trat nie eine Glucosurie auf. Nach 18-tägiger Prednisolonbehandlung war der Nüchternblutzucker wieder normal, eine Beobachtung, die schon von Lazarow (1952) und Fratroxson et al. (1953) gemacht wurde; diese Autoren wiesen auf den vorübergehenden Charakter des „Steroid-Diabetes" bei der Ratte trotz Fortsetzung der Corticoidtherapie hin.

Dìe Erhöhung des Seruminsulins ist jedoch bei allen Prednisolon-behandelten Gruppen offensichtlich. Einige Autoren führen den Glucocorticoid-induzierten $\mathrm{Hy-}$ perinsulinismus auf die damit einhergehende Hyperglykämie zurück (PERLITY und KIPNIS, 1966; WILLIAMsos, 1962). Bei unseren Versuchen aber wird der Anstieg von Insulin im Blut nicht unbedingt von höheren Blutzuckerwerten begleitet. Dies läßt sich bei $B e$ trachtung der Verhältnisse im Nüchternzustand leicht erkennen: Im Vergleich zur Kontrollgruppe steigt beim 3- und 18-Tage-Versuch der Insulinspiegel im Blut um 229\% resp. $257 \%$ an, obwohl statistisch keine signifikante Veränderung des Nüchternblutzuckerwertes nachzuweisen ist. Um die Wechselbeziehung zwischen Blutzucker und Seruminsulin nach Glucosebelastung (d.h. die 5-, 15-, 30-, 45- und 60-MinutenWerte) zu interpretieren, haben wir die Korrelation dieser beiden Parameter berechnet. Nachdem das Vorliegen einer Korrelation zwischen Blutzucker and Seruminsulin bei allen vier Versuchsgruppen statistisch gesichert war (Abb. 2, Tabelle 2), wendeten wir einen $F$-Test an, um zu prüfen, ob die erhaltenen Regressionen auseinander lagen. Auf diese Weise konnten wir feststellen, daß die Regressionslinie des 18-Tage-Versuchs über derjenigen der Kontrollgruppe lag ( $F=$ $8.59 ; p<0.001$ ), was ein sicherer Hinweis dafür ist, daß beim gleichen Blutzuckerspiegel nach 18-tägiger Prednisolonbehandlung mehr Insulin vorhanden ist als bei den Kontrolltieren. Für die 3- und 7-Tage-Versuche konnte dieser Nachweis nicht erbracht werden. Bei einem ihrer Versuche mit Chinesischen Hamstern bemerkten CambexL et al. (1966) ebenfalls, daB die Cortisontherapie zu einer wesentlichen Erhöhung des Plasmainsulins, jedoch nicht zu einem eindeutigen Anstieg des Blutzuckers führte.

Malaisse et al. (1967) deuten die Ergebnisse, die sie bei ihren in vitro-Versuchen mit Rattenpankreasgewebe erhalten haben, dahingehend, daß durch Glucocorticoidbehandlung die Empfindlichkeit des Insulin-Sekretionsmechanismus der beta-Zelle gegenüber Glucose verstärkt wird. Die Auswirkung der Prednisolonbehandlung auf die Reaktivität des endokrinen Pankreas gegenüber Glucose - d, h. die Fähigkeit, auf eine bestimmte Erhöhung des Blutzuckers mit einem Anstieg der Insulinsekretion zu reagieren -, haben wir durch Vergleich der Regressionskoeffizienten untersucht (Abb. 2, Tabelle 2). Dabei zeigte sich, daß zwischen den Regressionskoeffizienten der behandelten Gruppen und denjenigen der Kontrollgruppe kein 
statistisch signifikanter Unterschied besteht (3-TageVersuch; $p>0.2 ; 7$-Tage-Versuch: $p>0.3 ; 18$-TageVersuch; $p>0.2)$. Die Prednisolonbehandlung führte
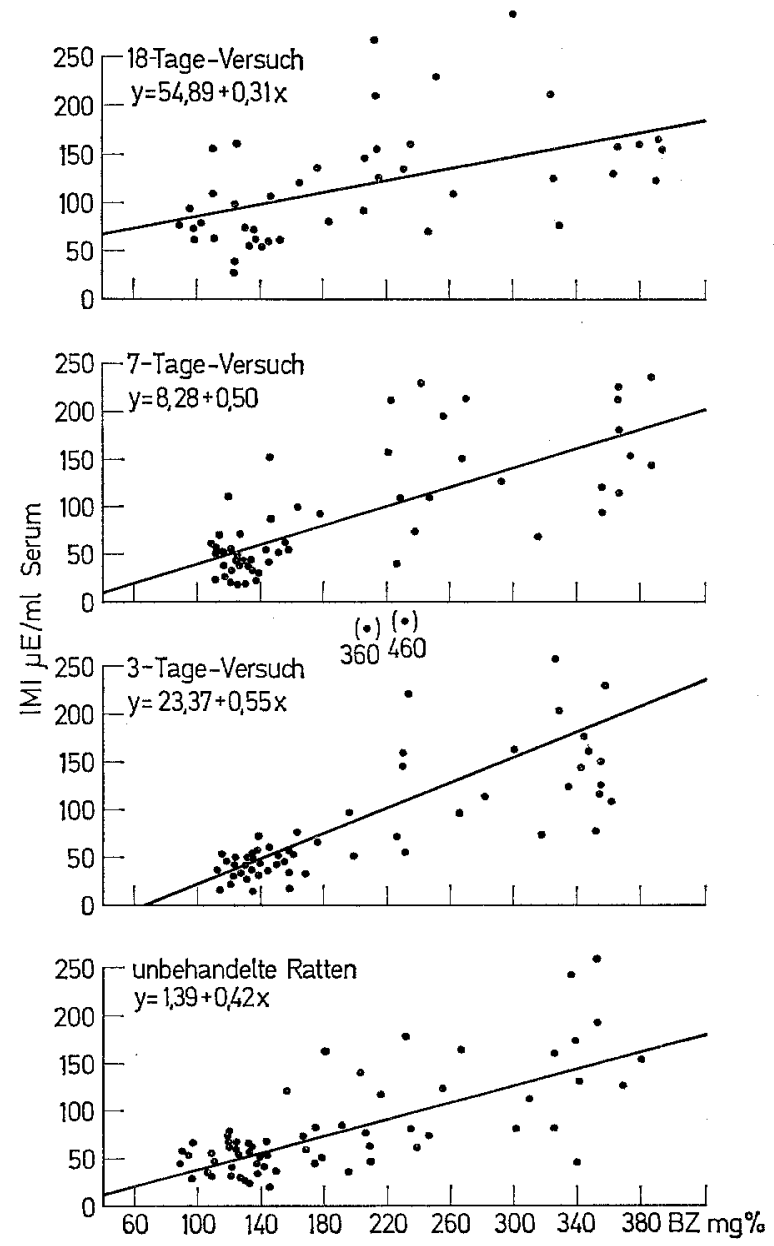

Abb. 2. Regressionsgeraden zwischen Blutzucker und Seruminsulin nach i.v. Glucosebelastung $(50 \mathrm{mg} / 100 \mathrm{~g}$ Körpergewicht), berechnet aus den 5-, 15-, 30-, 45- und 60-Minuten-Werten

Tabelle 2. Korrelationen zwischen Blutzucker und Seruminsulin nach i.v. Glucosebelastung $(50 \mathrm{mg} / 100 \mathrm{~g}$ Körpergewicht), berechnet aus den 5-, 15-, 30-, 45- und 60-MinutenWerten. a: Ordinatenabstand; $b$ : Regressionskoeffizient; $r$ : Korrelationskoeffizient

\begin{tabular}{|c|c|c|c|}
\hline Gruppe & a & $\mathrm{b} \pm \mathrm{SD}$ & $\mathbf{x}$ \\
\hline $\begin{array}{l}\text { Unbehan- } \\
\text { delte Ratten }\end{array}$ & -1.39 & $0.42 \pm 0.052$ & $0.7124(p<0.001)$ \\
\hline $\begin{array}{l}\text { 3-Tage- } \\
\text { Versuch }\end{array}$ & -23.37 & $0.55 \pm 0.103$ & $0.5692(p<0.001)$ \\
\hline $\begin{array}{l}\text { 7-Tage- } \\
\text { Versuch }\end{array}$ & -8.28 & $0.50 \pm 0.060$ & $0.7438(p<0.001)$ \\
\hline $\begin{array}{l}\text { 18-Tage- } \\
\text { Versuch }\end{array}$ & 54.89 & $0.31 \pm 0.081$ & $0.5009(p<0.001)$ \\
\hline
\end{tabular}

somit bei unseren Versuchen zu keiner statistisch signifikanten Änderung der Reaktivität des Pankreas gegenüber i.v. injizierter Glucose.
Zusammenfassend können wir aus den Ergebnissen unserer Untersuchungen feststellen, da $\beta$ bei der Ratte nach Prednisolonbehandlung im Vergleich zu den Kontrollen sowohl im Nüchternzustand als auch nach Glucosebelastung mehr Insulin im Blut nachzuweisen ist. Dieser Hyperinsulinismus scheint keine Folge einer Hyperglykämie zu sein und wird weder von einer Verminderung der Glucoseassimilation, noch von einer verstärkten Reaktivität des Pankreas gegenüber Glucose begleitet. Da es keinen Beweis für eine direkte Stimulation der Glucocorticoide auf die beta-Zellen gibt (Matatsse et al., 1967) und auch der Insulin-Abbau durch diese Hormone nicht beeinflußt wird (BERsow et al., 1957; ElgEE u. WrLLIAMs, 1955), kann man einen peripheren Antagonismus zwischen den Glucocorticoiden und dem Insulin vermuten, der, um den Kohlenhydratstoffwechsel im Gleichgewicht zu halten, zu einer vermehrten Insulinsekretion führt. Eine Stimulation der Gluconeogenese (LoNG et al., 1940), eine Hemmung der peripheren Phosphorylierung der Glucose (Astmore, 1964) sowie eine Behinderung der Glucoseaufnahme von Muskel- und Fettgewebe infolge verstärkter Freisetzung von Fettsäuren (FFA) (RAN DuE et al., 1963), stehen dagegen als Ursache dieses Antagonismus zur Diskussion. Auch die Kaliumdepletion kann dabei eine Rolle spielen (Krnselu et al., 1953).

Die statistische Auswertung wurde freundlicherweise von Herrn Dipl. Math. Böнms (Deutsches Rechenzentrum, Darmstadt) durchgeführt.

Fräulein JuTTA KRUsCHkE danken wir für ihre tatkräftige Mithilfe.

Sonderdruckanforderungen sind zu richten:

An die Bibliothek des Zentrums für Innere Medizin der Universität Ulm, Medizinisch Naturwissenschaftliche Hochschule, 7900 Ulm a.d. Donau, Steinhövelstraße 9.

\section{Literatur}

Asmmore, J.: The effects of glucocorticoids on insulin action. Diabetes 13, 349-354 (1964).

BASSET, J.M., and B. WALIACE: Influence of cortisol on plasma insulin in the sheep. Diabetes $16,566-571$ 1967).

BersoN, S.A., R.S. YALOW, and B.W. VolK: In vivo and in vitro metabolism of insulin- $\mathrm{I}^{131}$ and glucagon- $\mathrm{I}^{131}$ in normal and cortisone treated rabbits. J. Lab. clin. Med. 49, 331-342 (1957).

CAMPBeli, J., K.S. Rastogr, and H.R. HaUSteR: Hyperinsulinemia with diabetes induced by cortisone, and the influence of growth hormone in the Chinese hamster. Endocrinology 79, 749-756 (1966).

Conard, V.: Mesure de l'assimilation du glucose. Bases théoriques et applications cliniques. Acta med. belg. Ed. Bruxelles 1955.

ElGme, N.J., and R.H. WILLTAMs: Pituitary and adrenal influences on insulin- $\mathrm{I}^{131}$ degradation. Amer. J. Physiol. 180, $9-12(1955)$.

Franckson, J.R.M., W. Gerts, P.A. Bastenie, V. ConARD, N. CoRdirR et L. Kovacs: Observations sur le diabète stéroide expérimental du rat. Acta endocr. (Kbh.) 14, 153-169 (1953).

INGLE, D.J.: The production of glycosuria in the normal rat by means of 17-hydroxy-11-dehydrocorticosterone. Endocrinology 29, 649-652 (1941). 
-, and G. W. THore: Comparison of effects of 11-desoxycorticosterone acetate and 17-hydroxy-11-dehydrocorticosterone in partially depancreatized rats. Amer. J. Physiol. 132, 670 (1941).

KaRAM, J.H., G.M. GRODSKY, and P.H. ForshaM: The relationship of obesity and growth hormone to serum insulin levels. Ann. N.Y. Acad. Sci. 131, 374-387 (1965).

KINASH, B., and R.E. HarsT: Effect of ACTH and of cortisone on islets of Langerhans and pancreas in intact and hypophysectomized rats. Amer. J. Physiol. 178, $441-444$ (1954).

KInsEli, L. W., H.E. BaLCH, and G.D MrchaEls : Modification of "steroid diabetes" by potassium. Metabolism $2,421-423$ (1953).

KuINK, D., and D. EsTrich: Plasma insulin concentration in Cushing's syndrome and thyrotoxicosis. Clin. Res. 12,354 (1964).

LAzAROW, A.: The relation of glutathione to the diabetogenic effect of adrenal steroids. Diabetes 1, 171-182 (1952).

LoNG, C.N.H., B. KATZIN, and E.G. FRY: The adrenal cortex and carbohydrate metabolism. Endocrinology 26, 309-344 (1940).

Mataisse, W.J., F. Malatsse-Lagae, E.F. McCRaw, and P.H. WRIGHT: Insulin secretion in vitro by pancreatic tissue from normal, adrenalectomized and cortisol treated rats. Proc. Soc. exp. Biol. (N.Y.) 124, $924-928$ (1967).

Metant, F., H. Ditschuneit, K. M. Bartelt, H. FriedRIOH u. E.F. PFETFFER. Über die radioimmunologische Bestimmung von Insulin im Blut. Klin. Wschr. 43, 1000-1007 (1965).

NoweLL, N. W., and R.J. HowLaNd : The control of blood sugar in the laboratory rat and golden hamster. I. Intravenous glucose tolerance tests. Acta endocr. (Kbh.) 52, $149-153$ (1966).
PERT.fT, M., and D.M. KIPNIS: Effect of glucocorticoids on plasma insulin. New Engl. J. Med. 274, 1237-1241 (1966).

Randie, P.J., P.B. Garland, C.N. Halms, and E.A. Newsholme: The glucose fatty-acid cycle. Its role in insulin sensitivity and the metabolic disturbance of diabetes mellitus. Lancet 1963 I, $785-789$.

SCHWARZ, K., K.F. W TINGHS, K.P. Eymer u. Kopetz: Das Verhalten der insulinähnlichon Aktivität im Blut bei Patienten mit einem Cushing-Syndrom während einer Glucose-Doppelbelastung. Verh. deutsch. Ges. inn. Med. 68, $289-292(1962)$.

SutTer, B. CH.J.: Surrénales et insuline plasmatique chez le rat à jeun. Path. Biol. 12, 550-557 (1967).

VoLK, B. W., and S. S. LAZARUS: Ultramicroscopic studies of rabbit pancreas during cortisone treatment. Diabetes 12, 162-173 (1963).

VRANIC, M.: Effect of cortisol in guinea pigs with normal and atrophic exocrine pancreas. Diabetes 14, 194-200 (1965).

WEST, K.M.: Comparison of the hyperglycemic effects of glucocorticoids in human beings. Diabetes 6, 168-175 (1957).

WILLIAMson, J.R.: Studies on the effects of steroids on adipose tissue and beta cells. Fed. Proc. 21, 203 (1962).

ZuckER, H.D. : Alloxan sub-diabetes in rabbits detected by modification of glucose tolerance by adrenal cortex extract. Proc. Soc. exp. Biol. (N.Y.) 71, 597-601 (1949).

Dr. J. Marco, Doz. Dr. F. Metani, Dr. R. Goberna, Dr. W. H. RotT, Prof. Dr. E. F. PFeIfFer, Abt. für Endokrinologie u. Stoffwechsel des Zentrums für Inn. Medizin der Univ. $79 \mathrm{UTm}$

Steinhövelstr. 9 